\title{
Brookhaven's heavy ion collider goes live
}

[LONG ISLAND] Physicists at the Brookhaven National Laboratory on Long Island last week sent gold ions around the first ring of the Relativistic Heavy Ion Collider (RHIC), a $\$ 600$ million nuclear-physics facility built to create and study quark-gluon plasma.

Next, the RHIC team will send ions in the opposite direction around a second, $3.8-\mathrm{km}$ circumference, ring, opening the way for the facility's experimental programme to start this November.

The programme is the culmination of a two-decade quest by US nuclear physicists for an experiment powerful enough to explore the behaviour of quarks and gluons when nuclei collide at energies high enough to wrest these particles from their usual status as the constituents of protons and neutrons.

The tunnels that carry RHIC's rings, as well as some of the equipment which will feed ions into them, were constructed 20 years ago as part of a particle physics experiment, called Isabelle, to study proton collisions. But in 1983, high-energy physicists abandoned Isabelle for the more ambitious Superconducting Super Collider - itself abandoned by Congress ten years later.

The demise of Isabelle led Nick Samios, who had become director of Brookhaven in 1982 , to work with nuclear physicists to create a heavy-ion collider. Samios, who was ousted as director of Brookhaven two years ago, can now watch his dream reach fruition as an ordinary physicist working at the laboratory.

RHIC will serve as the main focal point for the Brookhaven laboratory, which has been in turmoil following a leak of radioactive tritium from the fuel storage tank of its research reactor. The reactor has been closed since the leak was discovered in 1997 (see box).

Almost 1,000 investigators will use the four detectors at RHIC - two large and two small — to study the character of the quark-gluon plasma. This plasma is expected to be created when gold ions collide at combined energies of $40 \mathrm{TeV}$, or $200 \mathrm{MeV}$ for each proton or neutron involved in the collision.

The detectors will use differing methods to detect the byproducts of the collision, reflecting uncertainty about what clues will betray the existence or the nature of the plasma. "We're talking about the interaction of hundreds of quarks and gluons," says Samios. "There's lots of speculation about what will happen. The problem is that you don't know what you are looking for."

Broadly speaking, one of the large detectors, called Phenix, will identify rare by-products of the plasma explosion, such as the $\mathrm{J} / \psi$ meson, while the other, Star, finds the tracks of the more common ones. Phenix has cost about $\$ 90$ million and Star $\$ 70$ million; each involves about 400 investigators.

Two more specialized detectors, Brahms

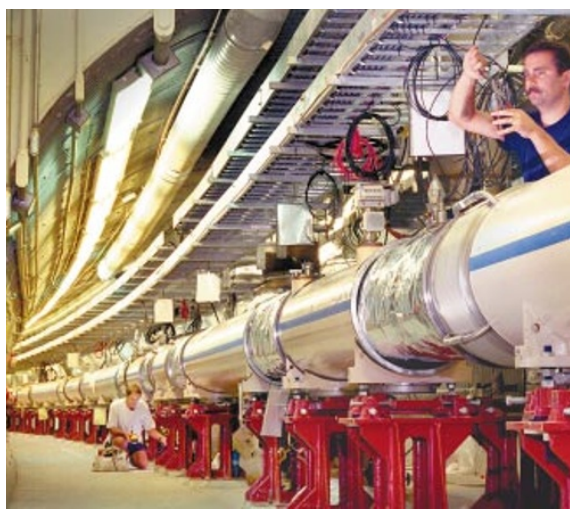

Collision course: rings have been placed inside a tunnel intended for ill-fated Isabelle accelerator.

and Phobos, each involve 50 investigators and cost about $\$ 8$ million.

Japan and Russia have been heavily involved in the design and construction of the RHIC detectors - their combined contribution is worth about $\$ 40$ million, according to project director Satoshi Ozaki. But there has been little European involvement. European

physicists are planning their own experiment, called Alice, using the Large Hadron Collider when it opens in six years' time at the European Laboratory for Particle Physics (CERN).

The $200 \mathrm{MeV}$ energy for each nucleon in a collision at RHIC is ten times the energy of the most powerful such experiment so far conducted at CERN, and four times the minimum energy that should smash the colliding nuclei into a plasma of quarks and gluons. Physicists are confident that plasma will be created at RHIC, but unsure of the characteristics of the transition to plasma. "The phase transition is a subtle thing to observe," says Tom Ludlam, associate director of the project.

The rewards of finding the plasma, however, are great. RHIC could recreate the conditions thought to characterize the Universe for the first fraction of a second of its existence, before quarks and gluons coalesced into protons and neutrons. The discovery of the plasma would confirm an important prediction of quantum chromodynamics, and its characterization could give a new insight into the nature of matter.

ColinMacilwain

\section{Leading a laboratory out of the mire}

[LONG ISLAND] The man trying to steer Brookhaven National Laboratory out of the tempest that consumed it when the local community reacted furiously to a radioactive leak from the laboratory is John Marburger, former president of the nearby State University of New York at Stony Brook

(Nature 386, 3; 1997).

Marburger is a softspoken administrator whose style contrasts to that of Nick Samios, the brilliant, ponytailed physicist who led Brookhaven for 15 years. Samios, and the university consortium which managed the lab for the Department of Energy, departed soon after the discovery of the leak.

In management terms, "this laboratory experienced a catastrophe in 1997," says Marburger. "The quality of science in the lab was so good that the Department of Energy was willing to accept an obsolescent management style. People were oblivious to the actual state of the management systems here."

With much of the day-today management of science delegated to Peter Paul, the deputy director, Marburger is concentrating on the lab's external relations, which he says are improving.

But a decision to restart the High Flux Beam Reactor one of three important user facilities at Brookhaven, closed since the discovery of the leak from a basin storing its spent fuel - is not expected until early next year, or later. 'It's a year later than I expected, and I'm disappointed in the schedule," says Marburger.

Given the opposition of local congressman, Michael Forbes (who this week switched from Republican to Democrat), and tight budgets, Marburger finds it hard to muster much optimism that the reactor will reopen at all, though "it is hard to imagine it not reopening, because it is so badly needed".

Since Brookhaven Science Associates (BSA), a new contractor involving the research consultancy Battelle, and Stony Brook, took over operation of Brookhaven last year, rumours have circulated that Battelle will cut staff and steer the lab towards technology which it can sell.

Marburger denies that either is being considered. "Battelle is delighted to be associated with the basic science at this lab," he says, adding that a shift to technology "would be a disaster. As president of BSA I intend to maintain the fundamental characteristics of this laboratory."

As for staff, Marburger

says the lab needs to expand its 3,100 staff, to generate the research income needed to cover its overheads. "'d like to increase the staff to 4,000 ," he says.

"We believe our basic science capability is not being fully exploited," Marburger says. "Our basic strengths are in fields that are a little old-fashioned." He declines to identify which fields, but says that growth areas should include structural biology, imaging technology, chemistry and materials science. 